Corrigendum

\title{
Corrigendum to "Peritumoral EpCAM Is an Independent Prognostic Marker after Curative Resection of HBV-Related Hepatocellular Carcinoma"
}

\author{
Xiao-Meng Dai, ${ }^{1}$ Tao Huang, ${ }^{2}$ Sheng-Li Yang, ${ }^{1}$ Xiu-Mei Zheng, ${ }^{1}$ George G. Chen, ${ }^{3}$ and \\ Tao Zhang \\ ${ }^{1}$ Cancer Center, Union Hospital, Tongji Medical College, Huazhong University of Science and Technology, Wuhan 430022, China \\ ${ }^{2}$ Department of Pediatrics, Tianyou Hospital, Wuhan University of Science and Technology, Wuhan, Hubei 430064, China \\ ${ }^{3}$ Department of Surgery, Prince of Wales Hospital, The Chinese University of Hong Kong, Shatin, New Territories, Hong Kong \\ Correspondence should be addressed to Sheng-Li Yang; jixufendou2013@gmail.com and Tao Zhang; taozhang66@outlook.com \\ Received 11 July 2017; Accepted 6 August 2017; Published 20 September 2017 \\ Copyright ( 2017 Xiao-Meng Dai et al. This is an open access article distributed under the Creative Commons Attribution License, \\ which permits unrestricted use, distribution, and reproduction in any medium, provided the original work is properly cited.
}

In the article titled "Peritumoral EpCAM Is an Independent Prognostic Marker after Curative Resection of HBV-Related Hepatocellular Carcinoma" [1], affiliation number one was given incorrectly. The corrected affiliation is shown above.

\section{References}

[1] X.-M. Dai, T. Huang, S.-L. Yang, X.-M. Zheng, G. G. Chen, and T. Zhang, "Peritumoral EpCAM is an independent prognostic marker after curative resection of HBV-related hepatocellular carcinoma," Disease Markers, vol. 2017, Article ID 8495326, 8 pages, 2017. 


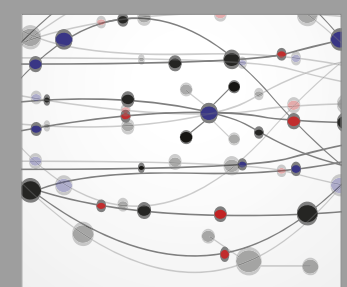

The Scientific World Journal
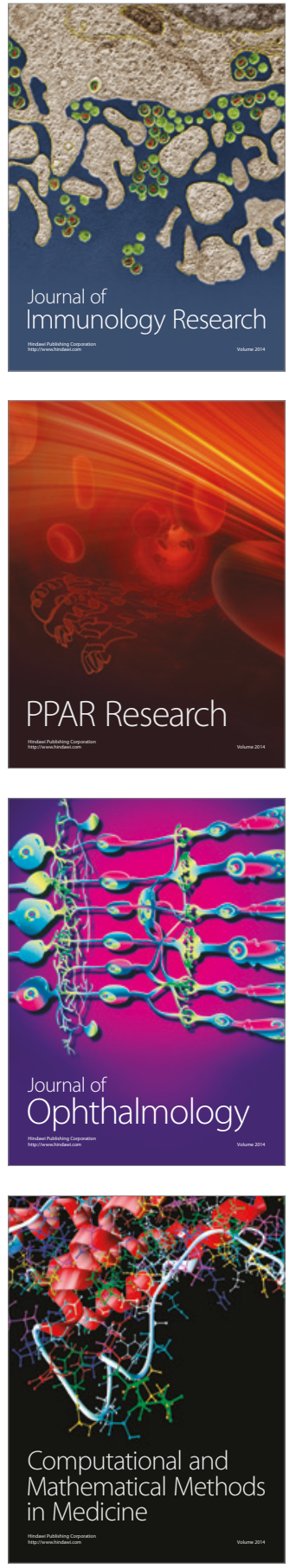

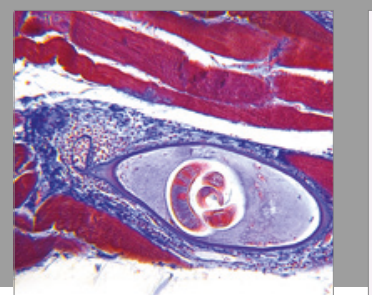

Gastroenterology Research and Practice
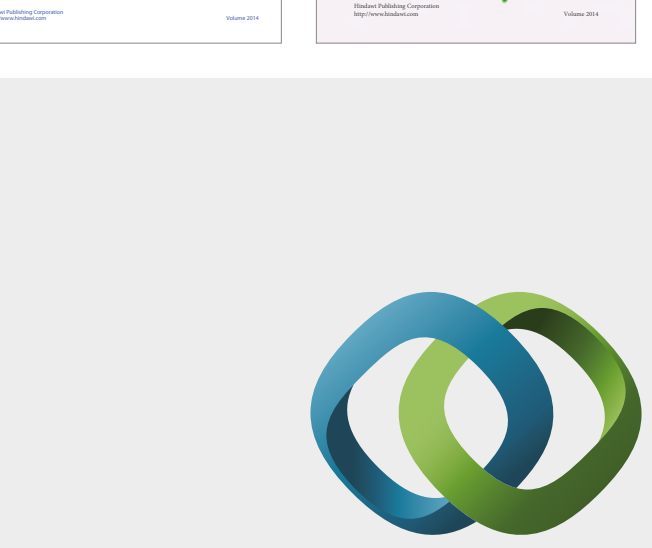

\section{Hindawi}

Submit your manuscripts at

https://www.hindawi.com
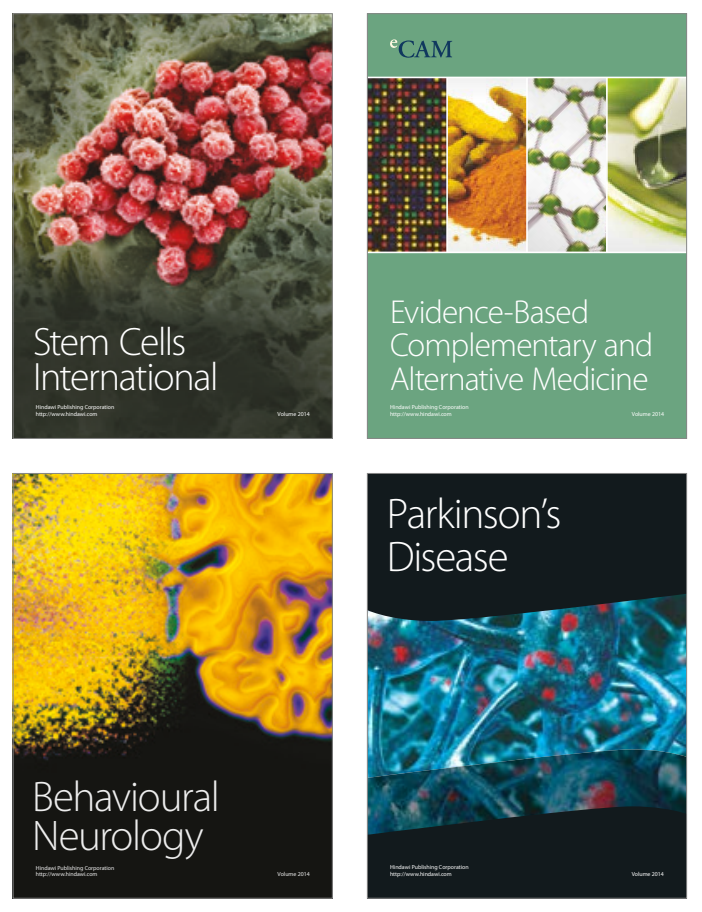
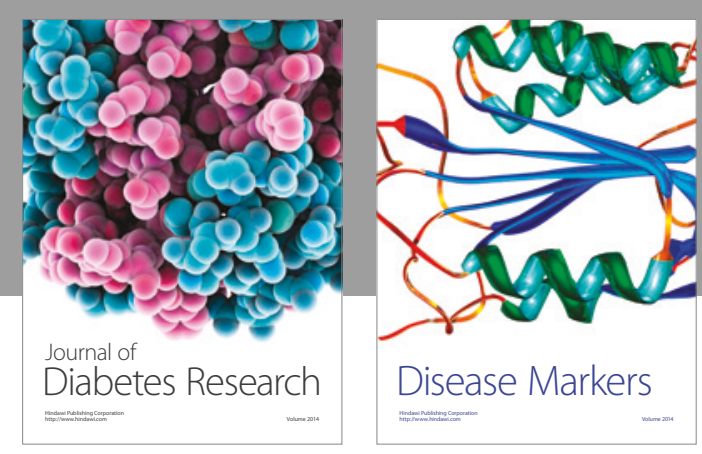

Disease Markers
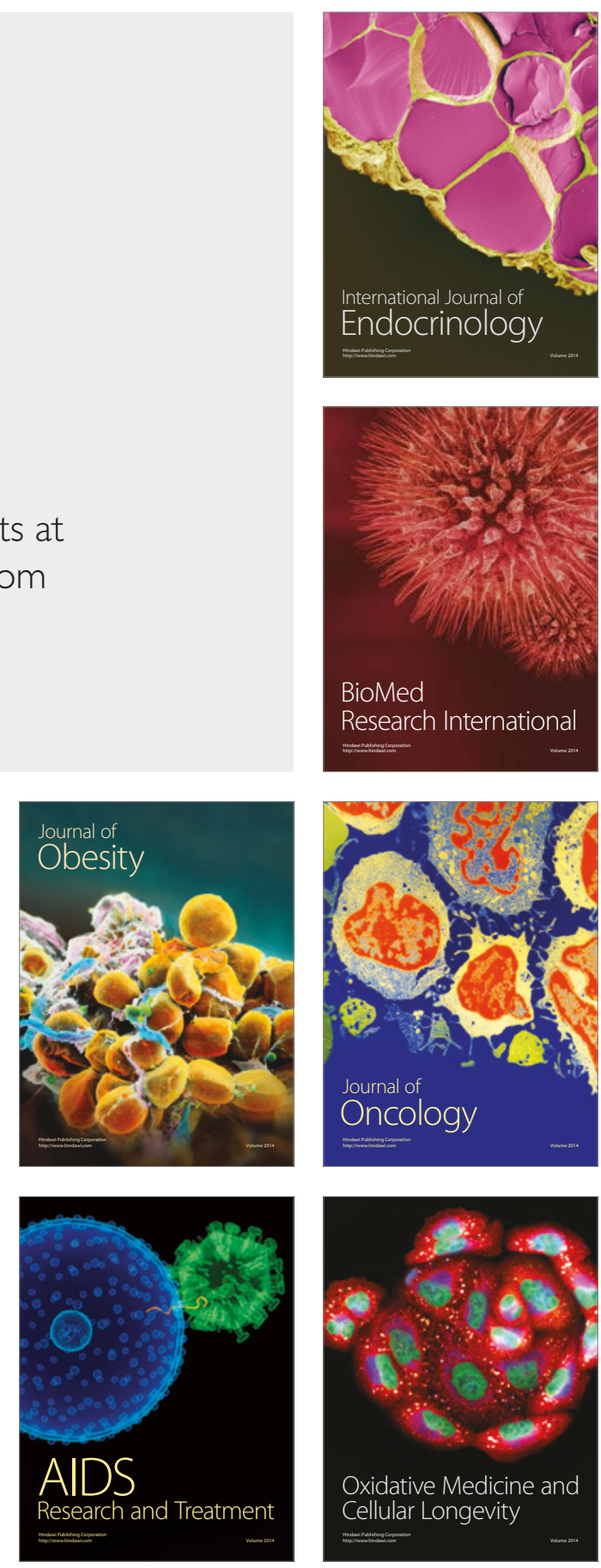